\title{
Enhancing Children's Language Learning and Cognition Experience through Interactive Kinetic Typography
}

\author{
Newman M. L. Lau ${ }^{1} \&$ Veni H. T. Chu ${ }^{1}$ \\ ${ }^{1}$ School of Design, The Hong Kong Polytechnic University, Hong Kong \\ Correspondence: Newman M. L. Lau, School of Design, The Hong Kong Polytechnic University, Hung Hom, \\ Kowloon, Hong Kong. Tel: 852-2766-4345. E-mail: newman.lau@polyu.edu.hk
}

Received: February 10, 2015 Accepted: April 7, 2015 Online Published: August 26, 2015

doi:10.5539/ies.v8n9p36

URL: http://dx.doi.org/10.5539/ies.v8n9p36

\begin{abstract}
This research aimed at investigating the method of using kinetic typography and interactive approach to conduct a design experiment for children to learn vocabularies. Typography is the unique art and technique of arranging type in order to make language visible. By adding animated movement to characters, kinetic typography expresses language meanings in a better and dynamic way. Kinetic typography may also help children to clearly grasp the relationship of difficult words by means of expressing, understanding and experiencing the cognition process. When children interact through the dictionary with animated typeface and movement, it brings on a more functional and profound impression on them for gaining knowledge. The present study takes children's development and learning theories into consideration by analyzing how they acquire information through interactive process to enhance their involvements. This study shows through the implementation of motion graphics, kinetic typography and information design, that a more diversified learning experience can stimulate and strengthen the sensitivity of children in their perception and learnability. It can also enhance children's understanding on the meaning of vocabularies, through kinetic expressions.
\end{abstract}

Keywords: language learning, kinetic typography, information design, interactive experience, motion graphics, engagement

\section{Introduction}

Learning should be an experience of discovering new things, rather than to be a pressure on students. Unfortunately, most of the students consider learning new things and studying as boring and unexciting. Nowadays, the Hong Kong government exercises an invisible pressure on the school students in their learning activities by conducting a number of tests, examinations and milestones to compete for achievements. Learning can actually be exciting, interesting and fun; but, it needs to be discovered by the students themselves, and it should not to be a 'spoon-fed education'.

\section{Background}

Years of research studies have conducted on the characteristics of children (Driver, Guesne, \& Tiberghien, 1985; Hohmann \& Weikart, 2002; Rogoff, Matusov, \& White, 1996). Studies that are directly related to the children's learning process are considered essential when designing the settings for children. Piaget proposed the following four stages of cognitive development: (i) sensorimotor stage, (ii) pre-operational stage, (iii) concrete operational stage, and (iv) formal operational stage (Krashen \& Tracy, 1982). However, these propositions are not usually put into practice. In each of these four stages, the learning modes of children remain different. Piaget believed that children, at a very early stage, begin to understand their world through simple and innate scheme and pattern. However, while they are growing up, these schemes and patterns tend to change (Papalia, Olds, Feldma, Martorell, \& Papalia, 2012). In this way, it is highly essential to consider the characteristics of children, when making the plan for setting up a teaching and learning process.

In the early eighties, Stephen and Tract developed a natural approach for children in learning, in order to make them get more involved in learning new things (Krashen \& Tracy, 1982). This approach aimed at making the learners as more relaxed as possible during the learning process. The approach is based on five hypotheses: (i) input hypothesis (ii) natural order hypothesis; (iii) acquisition learning hypothesis; (iv) monitor hypothesis, and (v) affective filter hypothesis. These five hypotheses are widely used in language teaching (Krashen, 1982). 
In order to make children interested in learning, it is first important to understand their needs, interests and preferences. Using this knowledge, we can implement a pedagogical proposal that children find meaningful and interesting. Previous studies have showed that, a deliberate approach to the selection and arrangement of materials may be taken to enhance young children's uses of literacy objects and related resources and thus provide young children with greater opportunities to engage in language and literacy interactions (Neuman, Koh, \& Dwyer, 2008). As suggested by Wu's study regarding to children's capacity for multi-signification, children would firstly produce pictographic symbols and then consistently transit them to the use of ideographic symbols for multi-signification in a consistent transition process, characterized with children's contingency association, focus variation, and extension chain (Wu, 2013). Interactive games and motivating activities have always been proved as effective tools to practise and reinforce new vocabularies for achieving better understanding of sentences (González, 2010). Pictures also function as important tools in stimulating children to forming hypotheses, predicting, sequencing and exercising memory.

The other main components of learning materials are words. They can be put into layouts through the means of typography. Its main purpose is communication, by the appearance of which it displays. For children's learning, the communication must appear in its most brief, simple and direct form (Tschichold, 1995). Kinetic typography can be applied to convey feelings, expressions, and explanation of a situation to a better way. It aims to reflect the informational composition of explaining the needs. Subtle expressions could also be represented by the help of small set of typographic variables, such as tempo, small movement, and size change (Shannon, Jodi, \& Suguru, 1997).

\section{Subject Domain}

The aim of this present research project is to design an interactive kinetic dictionary for the kindergarten students. The unique characteristic of this dictionary is to let the students interact with the words and vocabularies by observing and interacting with the movement and changes of words, color, and simple visual elements, so that they can better understand the meanings of the vocabularies, and deepen their memories as well.

In the following section, learning and cognition process is addressed. This highlights the major features from the children's perspectives that one should pay attention when preparing educational materials. The design approach on expression is then explained. It adapts to the design concepts phase by phase to develop their visual components. The two major parts includes typography and visual composition. Design with good choices of elements for typography gives a nice aesthetic and literal arrangement of visual elements. This enhances the perception of information. Enhanced visual composition with illustrations reinforces the cognitive understanding on expression and meaning. In the following section, kinetic typography is applied to create additional dimensions on time and dynamics on top of the static medium. Interactivity is also added to provide an initiative for children to practise and reflect their understanding. At the same time, this also serves as one of the key motivations and entertaining values during their learning experiences.

\section{Learning and Cognition Process}

The natural approach for language teaching was firstly developed by Terrell and Krashen (1982). In our case of learning vocabulary, it may regard this as a kind of learning new language information. We borrowed the approach as an unconscious process to make learning meaningful. The process involved the children to discover rules and relationships between various objects in order to acquire new knowledge based on the existing ones. This is called the acquisition/learning hypothesis.

Generally, children learn new things, more effectively with their interest and needs. The needs and interest of children aged 2-7 years usually involves simple entertaining elements. Whenever our design includes these features, it satisfies the fundamental requirement on this aspect. For example, gameplay elements with animated images are a great tool for learning because children feel relaxed and comfortable while playing games, and thus acquire new learnings more easily. Meanwhile, an ideological attitude to pedagogical innovations developed in a different sociocultural milieu could also be taken into considerations (Hu, 2002).

Cognitive development is directly related to the children's learning process. The target audience of this interactive dictionary is the children in the age group of 2-7 years, the pre-operational stage. In this stage, children begin to represent the world with words and images. Hence, by using symbolic thought, it will be more effective for them to learn languages (DeLoache, 1995a, 1995b; Uttal, Schreiber, \& DeLoache, 1995). Since they are fascinated by images and moving elements, learning vocabulary enables them to think about people, objects, and feelings. Teaching pre-operational children by using concrete teaching aids such as animated images related to the vocabularies that appear on the screen enables the children to interact with them. On the other hand, it is also very essential to provide a wide range of experiences to children through the learning processes such as 
providing them a learning path by which they can observe, discover and experience, in order to deepen their impressions in learning vocabularies.

The other part of Piaget's theory is about cognitive process. It includes three stages: (i) organization, (ii) adaptation, and (iii) equilibrium. Organization is the process of grouping and combining experiences with new and more advanced ways to understand new things, such as, vocabularies. When children come across new things, they try to organize the rationale from their previous experience or knowledge to matching and comparison, as much as possible, for understanding. The next stage is an adaptation process in developing, refining, and adjusting schemes to maintain equilibrium (Eggen \& Kauchak, 2007; Fetsco \& McClure, 2005). After the organization process, they acquire the new information, and then adapt it to their existing knowledge, maintaining it by assimilation or accommodation processes. In this way, these two processes work together, resulting in either disequilibrium or equilibrium in understanding the world.

In our design, the word "grow" appears to be a new vocabulary for children. "Grow" is in the process, which involves what is organized inside their existing knowledge of seed, leaves and flower. Children then adapt to the new information of growing into the relationship between seed, leaves and flower. After the accommodation process, this becomes their new knowledge. In our design, seed, leaves and flower are the key graphical elements to start with. The dynamics then include the animation, which visualizes the changes in appearance involving the biological stages from a seed to a leaf, and then finally becoming a flower. The animation can thus express and visualize the meaning of the vocabulary "grow" in such a way that it allows the children to understand easily.

\section{Design Approach on Expression}

\subsection{Typography}

Typography is the process of arranging visual elements, which includes letters, words and texts. On one hand, it is an art form of aesthetic medium. On the other hand, it is a scientific visualization of objects in space with precise numerical values (Dabner, Calvert, \& Casey, 2010). The fundamental elements of a letter can be further broken down into microscopic perspectives of size and weight, or even deconstructed into various stokes with different settings. With a variety of size and weight, the expression can be dramatically different. Moreover, different types of typefaces have different functions. For example, a bold and flat typeface, which is eye catching, could be used to attract the attention from the children. A bold typeface and character in a round shape with serif are also usually used, as children will be easily attracted to the bold character.

From the perspective of beauty and appreciation, the abstraction can be seen as a type of anatomy (Figure 1). This structure includes the construction of shapes and lines into forms and connected elements inside a letter, as a linguistic object. A combination of letters becomes a word. An elaboration from the arrangement includes spatial arrangement of individual letters to become a shape, which can be seen as a graphical form. In order to convey a message effectively, linking words to text can express the message and communicate it with the audience. A paragraph is to convey an idea by a connected meaning of words. The shape of a paragraph can be an attraction for the audience to read with a special visual order. The mixture includes the placement, within a page and over the pages, to guide the reading path of audience. Besides, typography is also participated in language-based meaning, which is the linguistic formation of text. The linguistic meaning of typography creates a communication media to audience, based on the sets of symbols for language including letters and words, and also space between words and punctuations.
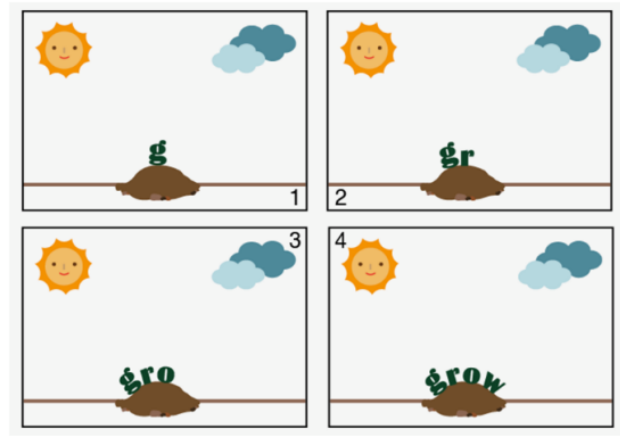

Figure 1. Abstraction as a type of anatomy 
The text not only communicates the specific meaning with a word itself, but also possesses its characteristics by the color or texture, and elevates the aesthetic point of view in a powerful way. A typeface of typography can express different feelings, and give different expressions (Figure 2). In order to express meanings and emotions through a text, we can apply color to the text to achieve special effects, or transform it as an image. The other ways to compose a text as an image is by symbolizing its characters, making use of the shape of character and imagine its shape to an image. For example, emoticon is a category of text icons, which tries to impose emotional expressions. Through this method, words can be transformed into non-textual meaning, thus expressing extra feelings through the words in its own right, as the images can be metamorphosed into types. One of the very common examples of the symbolization of word form emoticon is " $\mathrm{D}$ ". As the shape and form of " $D$ " is a semi-circle, it looks like a mouth of a smiling face.

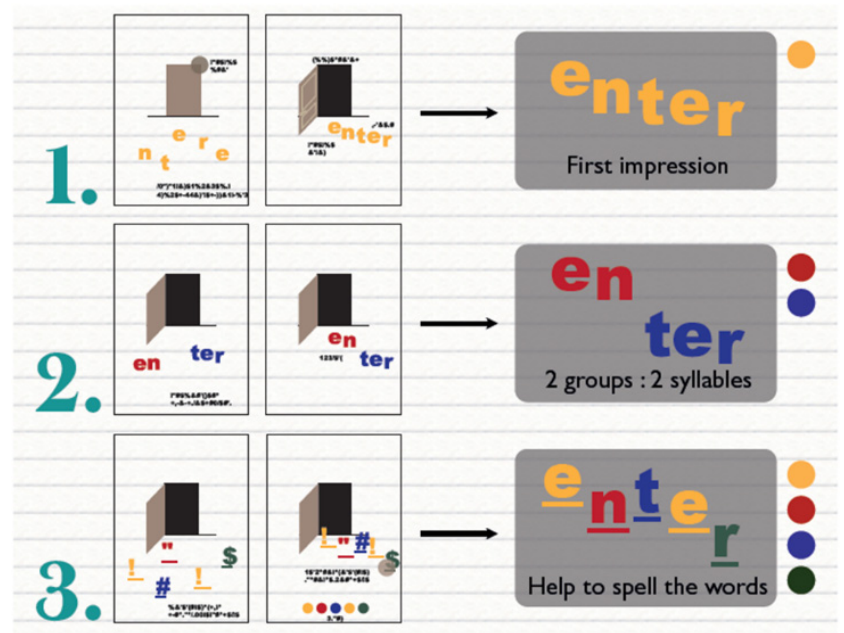

Figure 2. Typography to give characteristics and expressions

Moreover, colors also play an important role in expressing feelings and emotions. Different colors have their own universal characteristics. Besides, colors have their own temperature - cold and warm; for example, reds, oranges, and yellows can stimulate the sense. These colors give exciting feelings of cheeriness and aggressiveness as well. On the contrary, blues and greens impart the feeling of "coolness" and "relaxation", by bringing up the emotions of dispassionate, peace or disheartened. On the other hand, red is a common signal in colors to advance towards the viewers. In order to create a dynamic motivation, using high intensities of color can make this illusion to the viewers. Primary colors (Figure 3) can also attract audience. When applied into the design of children graphics, these colors can vary to become color palette (Figure 4) of expressions.

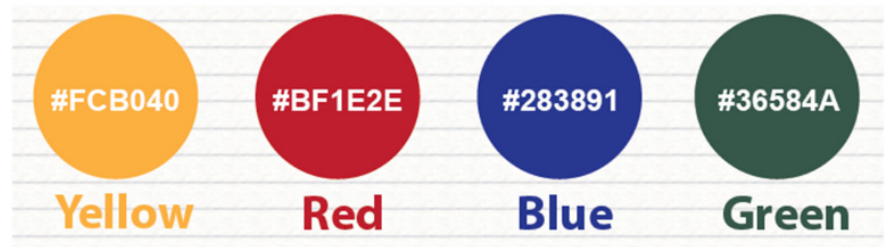

Figure 3. Primary colors (codes in \#RRGGBB) of attraction
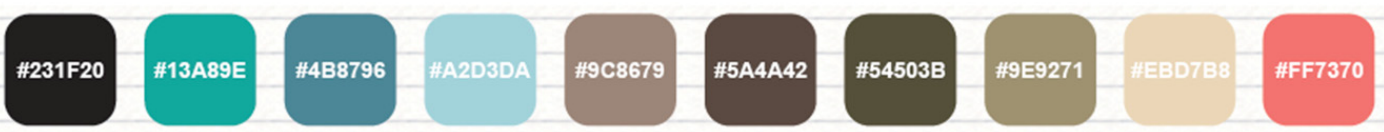

Figure 4. An example of color palette (codes in \#RRGGBB) 


\subsection{Visual Composition}

Colorful pictures and graphic illustrations can stimulate children's visual senses, in which they usually play an important role in making children interested or drawing their attention to a book. In this way, the meanings are successfully communicated through different combinations of texts and images. Since children are limited in words and vocabularies, images carry the important responsibility of communicating with them and engaging their thinking skills with the colorful and attractive pictures are inputting as very important elements inside a child's dictionary. Children love colorful pictures and different kinds of illustrations, as these can inspire their imaginations.

Good and correct use of images is particularly effective for the children to memorize, as well as helping them in their recognition processes, as compared to the function of pictures appearing in children's illustration books (Figure 5). In some cases, a direct visualization of an object, for instance, a tree, can serve one of the most effective ways for expressing the meaning. Visual effects or decorations can also be added to enhance the meaning of words. In order to convince children to read more, some playful and colorful decorations can be added to the text body (Figure 6).

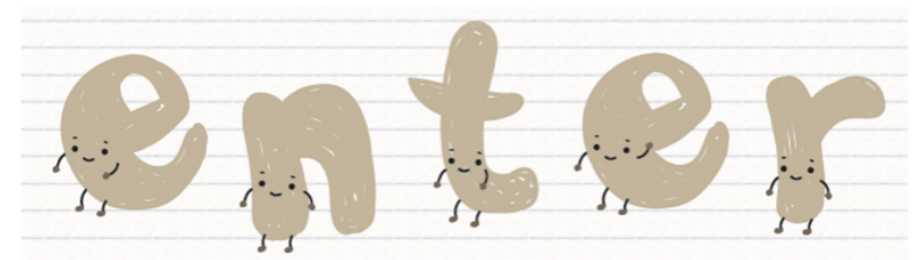

Figure 5. Illustration to attract children attention

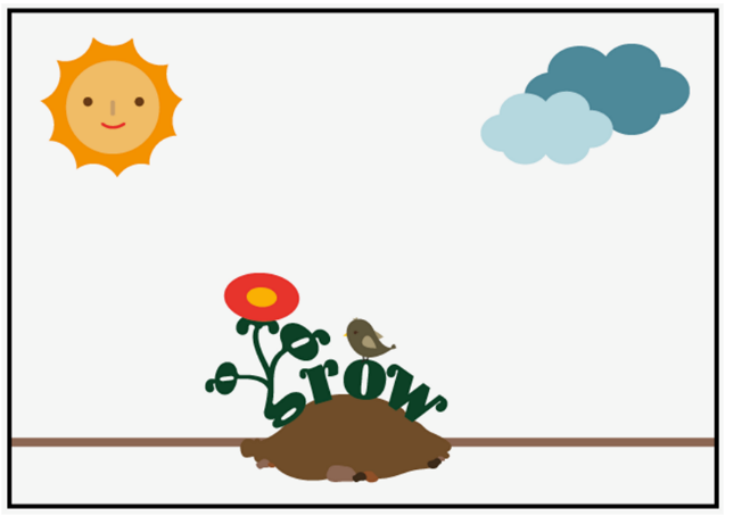

Figure 6. Use of decorative elements

Another effective way to express the meaning of vocabularies is to use texture on a character or word. Some kinds of things have its features on its texture. For example, we can identify different animals by observing their texture, skin and pattern. Similarly, a pineapple has a regular geometrical pattern with a pointy shape at the middle of each texture piece, and a zebra has a black and white stroke on its body. For some of the vocabularies, particularly those noun forms, patterns and textures can be used on its character, in order to express meanings for children.

\section{Execution on Kinetic Typography}

Kinetic typography (Figure 7) is the technical name for "moving text" by using video animation with mixing motion and text to express new ideas. It focuses on understanding the effect of time by the expression of text. The text will be presented over time in such a manner, so as to convey or evoke a particular idea or emotion. Its foundation comprises of structures and physical characteristics. Moreover, the arrangement is designed in space and becomes a layout. Through the time dimension, elements are moved within or beyond the boundary. Its dynamics is the major feature, which signifies the meaning of kinetic typography. 
Techical name of "Moving Text"

using video animation + mixing motion + text

express feelings, ideas and emotions.

focuses on understanding the effect time by the expression of text.

Figure 7. Interpretation of kinetic typography

\subsection{Fundamental Structure}

The fundamental structure of typography here includes the settlement of character, word and phrase. This connects to a text presentation, which further develops to facilitate the cognition in reading. Our human eyes are capable of reading text rapidly from word-to-word. This process is highly affected by the appearance of words; for example, size in space. It leads to a technique in the possibility of manipulating the design of typographic forms from time-to-time in expression information. Another important criterion to be considered while designing media for children is that they are of different characteristics in cognition of reading, as compared to the other stages of childhood or even adults. Eye movement is also considered in the kinetic typography; in which the movement of words can enhance readers' to read in a rhythmical form related to their eye movement. A successful reading path is to reduce the unnecessary eye movement of the reader, in order to create a clear and effective reading path to communicate with the readers, with the need to express the ideas and emotions more functionally. As an example in Figure 8, the character "g" gradually grows from the soil. This creates a clear reading path for the children to get attended and read.

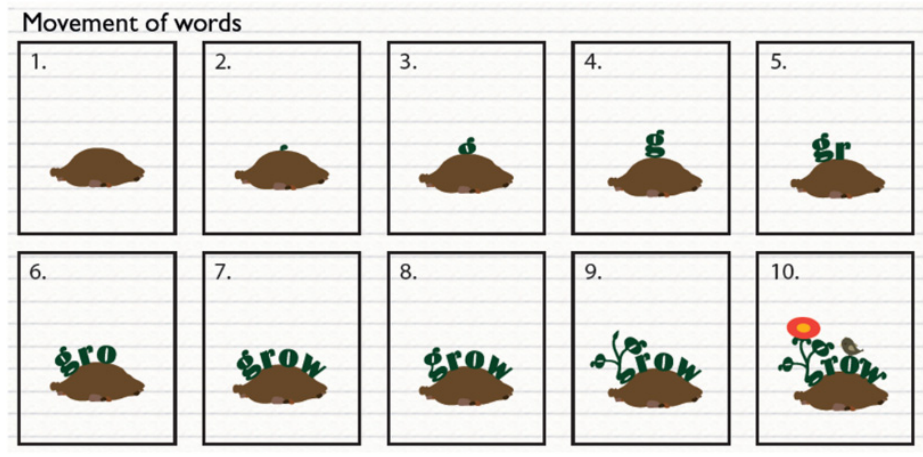

Figure 8. Movement of words growing up

After putting things together, composition acts as a visual structure for organizing and combining the distinct parts of words. It forms a whole holistic picture on the design of kinetic typography. Besides, a wide range of format can be applied, for example, two or three dimensional space, black and white space, negative and positive space, figure and ground, symmetrical and asymmetrical relationships, etc. In other words, it serves as an essential and effective visual communication, in terms of control and intended message. In negative and positive space, the function of the negative space is to support the positive "image" in a given area. While the white space can create tension and contrast for typography, it can also be used to highlight any important words. If there is only one word at the center of a white screen, the readers will focus on the word by using the white space around.

Motions play an important role in the kinetic typography, as kinetic typography involves time, rhythm, movement, speed, space, etc. Kinetic typography mainly focuses on the power of communication through the basis of time dimension. In order to make communication appear in a more effective way, making good use of the motion is very essential. From the experience, children can easily get attracted to the things that move around a space. For example, children, or even babies, like watching the television even though they cannot really understand what they see. The phenomenon behind this is that they are simply captured by the moving objects. From this aspect, moving words in a kinetic form gives an advantage to, first of all, draw the attention from the children. When the characters are arranged to appear or move in a pre-defined pathway, it can lead the reading 
path of children, apparently controlling their pace of learning. On the other hand, through the variation of movement, children can discover the meaning lying behind. As an example in Figure 9, the word "grow" can be characterized in animation based on the meaning of the word, the movement would start from the character " $\mathrm{g}$ ". The counter of "g" seems like a seed, and the loop of "g" looks like the root of a plant. The character "g" will then grow up, imitating the growth of a plant. The counter of "g" will grow into a small sapling, and then further grow into leaves and flower. Hence, this movement can let children observe and discover the meaning of words.

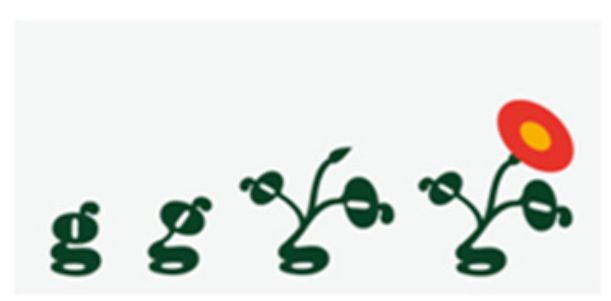

Figure 9. Animated character

\subsection{Interactivity}

Interactive gaming is one of the most effective tools for children to practise and reinforce the understanding in learning new things. Besides, games are also equally motivating and challenging, helping children to interact and get more involved in learning. To lower the challenge level, Figure 10 shows an example of hints by various colors. The children can base on the sequence of colors, point and drag the correct sequence of words to become the answer (Figure 11). The combination of game and vocabulary leaning can enhance children's interest in participation.

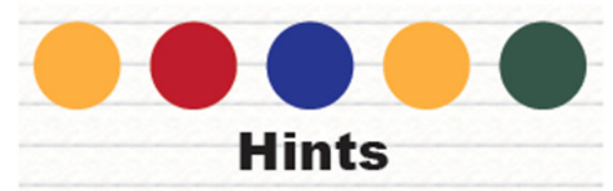

Figure 10. Sequence of words represented by color

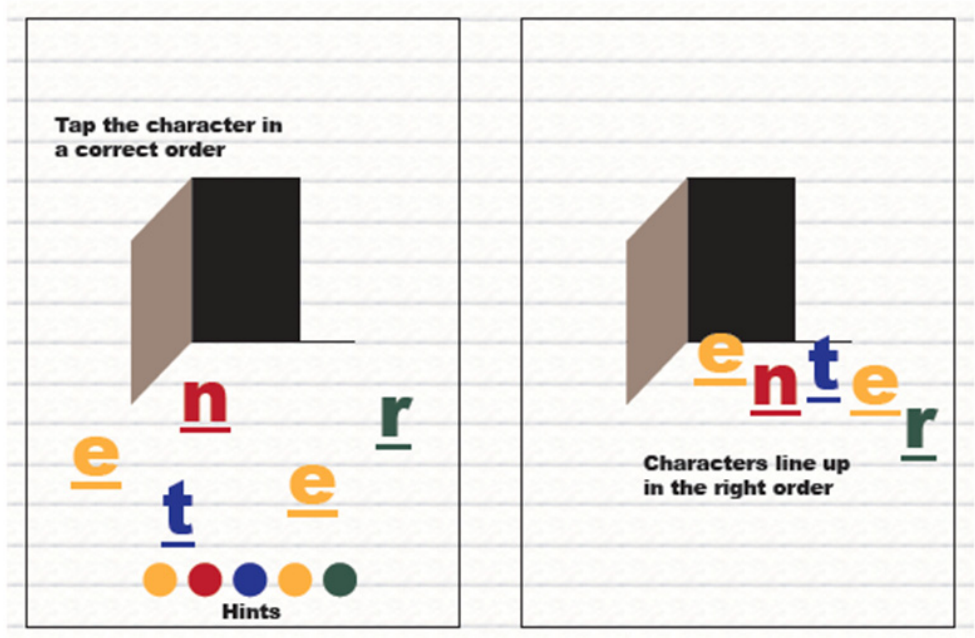

Figure 11. Click and drag to align the sequence 
In recent times, technology has enabled various interaction methods by means of different portable computer devices. One of the most common devices among these is the tabloid/tablet. One may find it amazing that most of the children nowadays are somehow able to use their fingers effectively to control what is on the screen. Together with our discussions and our design, we aimed to involve the implementation of user-interfaces on tabloids/tablets.

Based on the previously mentioned cognition process, children acquire new vocabularies through their experiences and observations. In the example of the word "grow", it comes with some simple button in the interface to lead them for the interactivity. The basic graphical elements include the seed, leaves and flowers. These elements appear on screen at the beginning, and then wait and trigger the children for input process. The Sun and clouds (Figure 12) appear in the interface, and children make use of the accommodation process to relate with the growing process of the plant. When children press on those elements, interactive events happen. The Sun shines on the seed; the clouds rain on the plant and thus fertilize the seed (Figure 13). After that, the seed gradually grows into leaves. This process enables the children to get an active involvement in the learning process. Such an experience makes them in understanding the process of growth and development.

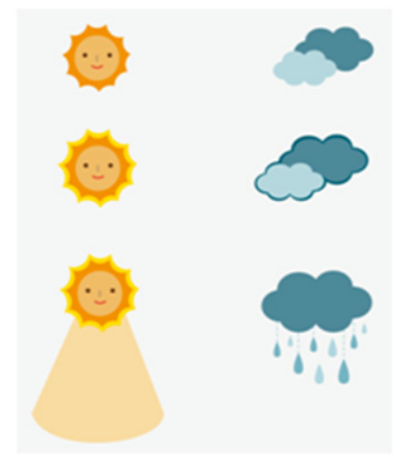

Figure 12. Graphical feedback to enhance understanding
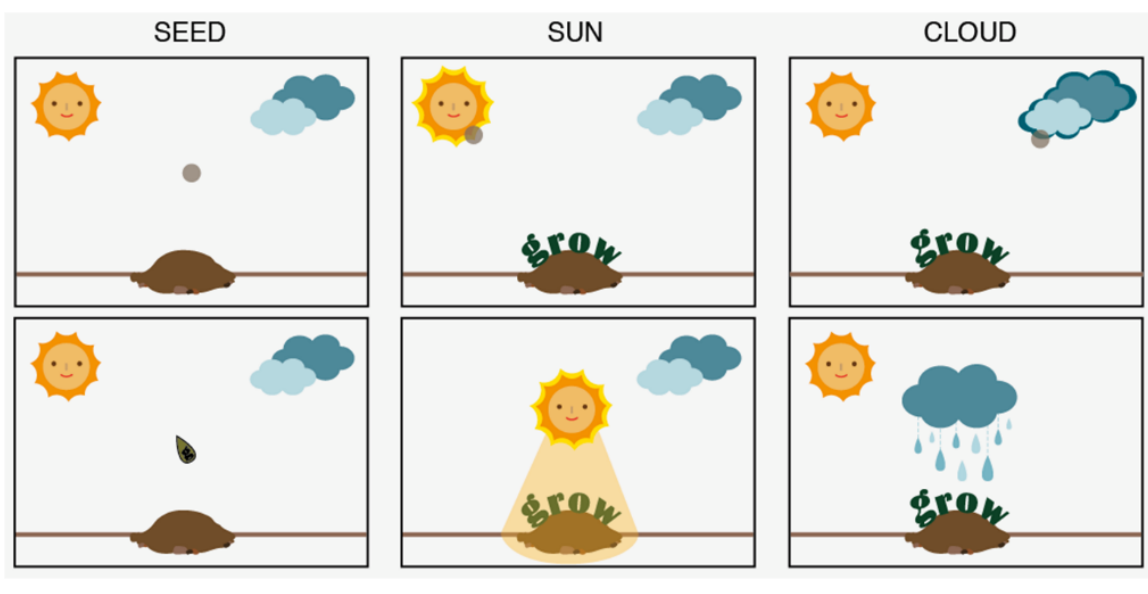

Figure 13. Different types of interactive input

\section{Conclusions}

This research study investigates the methods for children's language learning and cognition experience by using kinetic typography with interactive elements. The unique art form of typography and interaction design could eliminate the passivity of children, enhance their learning interests and cognitive activity. In the area of language learning, the cognition and memorization of vocabularies is essential and prepared as a beginning step for children aged 2-7. The motivation on the interest and interactivity for vocabulary learning should, at the same time, be considered.

The working structure for preparing an interactive kinetic dictionary for kindergarten students has been 
composed. This attempts to be a generic and working framework for enhancing the boring and unexciting learning experience and working around the pressures here in the region or in general. By adding animated movement to the characters, the vocabularies could be expressed in a better and dynamic way. The children's memories would be deepened by observing and interacting with the impressive characters. They could also have a better understanding on the meaning of the vocabularies as well as the relationship of difficult words through the design of expressions, which includes changes of words, colors and simple visual elements.

The learning and cognition process has been addressed. An effective method for preparing materials for teaching children to learn vocabularies through playing and interacting with the kinetic typography has been created. The design concepts of developing the visual components have been addressed phase by phase. Through the implementation of motion graphics, kinetic typography and information design, a more diversified learning experience can stimulate and strengthen the sensitivity of children in their perception and learnability. Further experiment can be attempted to investigate the ideological attitude of children under different sociocultural milieu.

\section{References}

Dabner, D., Calvert, S., \& Casey, A. (2010). Graphic design school: The principles and practices of graphic design. Hoboken, NJ: John Wiley \& Sons.

DeLoache, J. S. (1995a). Early symbolic understanding and use. In D. Medin (Ed.), The psychology of learning and motivation (pp. 65-114). New York: Academic Press. http://dx.doi.org/10.1016/S0079-7421(08)60372-2

DeLoache, J. S. (1995b). Early understanding and use of symbols. Current Directions in Psychological Science, 4, 109-113.

Driver, R., Guesne, E., \& Tiberghien, A. (1985). Children's ideas and the learning of science. In R. Driver, E. Guesne, \& A. Tiberghien (Eds.), Children's ideas in science (pp. 1-9). Buckingham: Open University Press.

Eggen, P., \& Kauchak, D. (2007). Educational psychology windows on classrooms. New Jersey: Prentice Hall.

Fetsco, T., \& McClure, J. (2005). Educational psychology: An integrated approach to classroom decisions. Boston: Allyn and Bacon.

González, N. I. P. (2010). Teaching English through stories: A meaningful and fun way for children to learn the language. PROFILE, 12(1), 95-106.

Hohmann, M., \& Weikart, D. P. (2002). Educating young children: Active learning practices for preschool and child care programs. MI: High/Scope Press.

Hu, G. (2002). Potential cultural resistance to pedagogical imports: The case of communicative teaching in China. Language, Culture and Curriculum, 15(2), 93-105. http://dx.doi.org/10.1080/07908310208666636

Krashen, S. (1982). Principles and practice in second language acquisition. New York: Pergamon Press.

Krashen, S. D., \& Tracy, D. T. (1982). The natural approach: Language acquisition in the classroom. Hayward, CA: Alemany Press.

Neuman, S., Koh, S., \& Dwyer, J. (2008). The child/home environmental language and literacy observation. Early Childhood Research Quarterly, 23, 159-172. http://dx.doi.org/10.1016/j.ecresq.2007.11.001

Papalia, D. E., Olds, S. W., Feldman, R. D., Martorell, G., \& Papalia, D. E. (2012). Experience human development. New York: McGraw-Hill.

Rogoff, B., Matusov, B., \& White, S. (1996). Models of teaching and learning: Participation in a community of learners. In D. Olson, \& N. Torrance (Eds.), The handbook of cognition and human development (pp. 388-414). Oxford, UK: Blackwell.

Shannon, F., Jodi, F., \& Suguru, I. (1997). Kinetic typography: Issues in time-based presentation. CHI 97, 269-270. http://dx.doi.org/10.1145/1120212.1120387

Tschichold, J. (1995). The New Typography. London: University of California Press.

Uttal, D., Schreiber, J. C., \& DeLoache, J. S. (1995). Waiting to use a symbol: The effects of delay on children's use of models. Child Development, 66(6), 1875-1889. http://dx.doi.org/10.2307/1131916

Wu, L. Y. (2013). Understanding children's concept formation and writing emergence from the perspective of graphical multi-signification: Evidence and pedagogical implications. Language, Culture and Curriculum, 26(3), 266-28. http://dx.doi.org/10.1080/07908318.2013.849719 


\section{Copyrights}

Copyright for this article is retained by the author(s), with first publication rights granted to the journal.

This is an open-access article distributed under the terms and conditions of the Creative Commons Attribution license (http://creativecommons.org/licenses/by/3.0/). 